\title{
Chaotic Behavior in a Flexible Assembly Line of a Manufacturing System
}

\author{
Mohammad Sajid \\ Mechanical Engineering Department \\ College of Engineering \\ Qassim University \\ Buraidah, AlQassim, Saudi Arabia \\ msajid@qec.edu.sa
}

\author{
Fahad Almufadi \\ Mechanical Engineering Department \\ College of Engineering \\ Qassim University \\ Buraidah, AlQassim, Saudi Arabia \\ almufadi@qec.edu.sa
}

\author{
Mirza Jahanzaib \\ Mechanical Engineering Department \\ College of Engineering \\ Qassim University \\ Buraidah, AlQassim, Saudi Arabia \\ mirza@qec.edu.sa
}

\begin{abstract}
The purpose of the present work is to study the chaotic behavior in a flexible assembly line of a manufacturing system. A flexible assembly line can accommodate a variety of product types. Result analysis is performed to obtain time persistent data. The behavior of the system is observed for Work-In-Process, as assembling systems are sensitive during processing. It is found that the average Lyapunov exponent is positive in the considered case, and thus chaotic behavior may be present in flexible assembly lines.
\end{abstract}

Keywords-Flexible assembly line; Chaos; Lyapunov exponent

\section{INTRODUCTION}

Due to the rapid recent development in assembling technologies and advancement in assembling methods, there is a need to critically investigate, and understand, an assembly system's environment. The identification and prediction is also a fundamental task, not limited to the field of engineering but in all kinds of other fields as well. During the last few decades, chaos theory has been applied in manufacturing systems and some initial research was carried out to analyze chaotic behavior in [1]. Chaos in discrete production systems was investigated in [2] using nonlinear analysis and a dynamical approach was introduced in [3] for modeling and control of production systems, based on nonlinear dynamics theory.

It is known that chaos is the phenomena of occurrence of bounded non-periodic evolution in completely deterministic nonlinear dynamical systems with high sensitive dependence on initial conditions. The nonlinearity of a system is considered a necessary condition. Sometimes, chaos is called deterministic randomness and is associated with the impediments of forecasting. The chaotic behavior generates a kind of randomness and a loss of information about initial conditions, which might explain somewhat complex behavior in real systems. Moreover, a system that is chaotic has a long-term behavior that can be hard to predict or simulate. Using chaos theory, it is easier to control more difficult and complicated cases compared to using traditional methods which are being utilized for a long time in manufacturing.
Alfaro and Sepulveda [4] studied the dynamic behavior of a reactive system; a system where there is no determined schedule and the tasks of operations to machines are assigned according to the state of the system. This research was performed using discrete event simulation to represent the system and its analysis was done by using nonlinear dynamical systems theory. Further, some significant research works for the chaotic behavior in manufacturing have been conducted in [5-6]. Little more extension in this direction is also found for the assessment of fidelity of control-theoretic models of WIP in autonomous work systems [7]. Furthermore, a nonlinear dynamics approach to model the complexity of a manufacturing system can be found in [8] whereas a nonlinear characterization of the performance of production can be found in [9]. An application of neural networks and vector support machines having a chaotic behavior is given in [10] for the forecasting of the time varying average number of parts in a waiting line of a manufacturing system.

The theoretical tool used for quantifying chaotic behavior is the notion of a time series of data for the real system [11]. The analysis of chaotic time series has been reviewed in [12]. The Lyapunov exponent is one important tool to define chaos. It is known that a system's behavior is chaotic if average Lyapunov exponent is positive [13]. The Lyapunov exponent, for a onedimensional time-series $\mathrm{k}$ data, is computed by:

$$
\lambda=\frac{1}{k} \ln \frac{d_{k}}{d_{0}}
$$

where $d_{0}=\left|x_{j}-x_{i}\right|, d_{k}=\left|x_{j+k}-x_{i+k}\right|$ and $x_{i}, x_{j}$ are two close values on the two different time series.

In order to detect chaotic behavior in a flexible assembly system, there is a need to describe the assembling process. In this research, Work-In-Process (WIP) of assembling system has been chosen to describe system behavior, as it imposes dynamism if not properly controlled [14]. A real flexible assembly line (with various performance variables) has been analyzed by means of process modeling software and the Lyapunov exponent. 


\section{METHODOLOGY}

Several researches have been done on chaos in manufacturing as mentioned in the introduction section, but the fundamental work is credited to Taken [15] who detected chaotic behavior in turbulence. The methodology adopted in our case is analyzing the system, obtaining a time series of assembly lines and observing chaotic behavior. The proposed algorithm is presented after reviewing the selection of the tool/technique for analysis.

In flexible assembling systems, parts are assembled in a respective station and there exists an interaction of parts with the machines. Apart from interaction, dominating relationship also exists in which a change in any parameter in the respective station affects the work in process in the subsequent stations. The system chosen for analysis consists of stations where different parts arrive and are assembled in a series of steps. Many approaches can be applied, for example analytic modeling, queuing theory and simulation. The analytical models demand too many assumptions while in queuing theory the estimates of expected value of the inter-arrival time distribution and service-time distribution are not exact. The queuing theory formula does not provide any information on the natural variability (dynamism) in the system [16]. On the other hand, simulation involves modeling of a process or system in such a way that the model mimics the response of the actual system to events that take place over time. A simulation run gives a number of variables in the output, including total production, average waiting time in queue, maximum waiting time in the queue, flow times, work in process, etc. Time persistent data of WIP is selected for the system. The mathematical form of WIP in [14] and calculated as:

$$
W I P=\frac{\left(\frac{M T B F-M T T R}{M T B F}\right)(Q)\left[n_{o}\left(T_{s u}+Q_{b} T_{c}+T_{n o}\right)\right]}{\left(\frac{n S_{w} H_{s} R_{p}}{n_{o}}\right)}
$$

where MTBF is the Mean Time Between Failures, MTTR is the Mean Time To Repair, Q is the Quantity actually produced, $n_{0}$ is the number of operations in the routing, $T_{\text {su }}$ is the setup time, $T_{c}$ is the cycle time per part; $T_{n o}$ is the non operation time, $Q_{b}$ is the batch quantity, $n$ is the number of machines, $S_{w}$ is the shifts per week, $H_{s}$ is the hours in a shift, and $R_{p}$ is the production rate.

The following algorithm is proposed for identifying the presence of chaos in a flexible assembly line:

(i) Finding a time series of the variable to be analyzed and the response obtained from the simulation model.

(ii) Taking the absolute difference of the series and obtain the logarithm of the difference values.

(iii) Reconstructing the variables for describing behavior using separation distance plots and measuring the difference for each data values. (iv) Estimating the sensitivity by the Lyapunov exponent; if it is greater than zero, then chaotic behavior exists in the system.

The algorithm is implemented on an assembly system that consists of a series of steps where parts are passed through decision process as described in subsequent section.

\section{CASE OF A FLEXIBLE LINE (PROCESS DESCRIPTION)}

The arriving parts are cast metal bodies that have already been machined to accept electronic parts. There are two parts, $\mathrm{A}$ and $\mathrm{B}$, produced in adjacent departments (out of bound for this model). Part A arrives in the system with a mean time of 5 minutes. Part A is prepared where mating faces of the cases are machined, deburred and cleaned with a combined process time of 6.5 minutes. After the preparation, the work part is transferred to the sealer. The work part B arrives in batches of four units and the time between the arrival of successive batches has a mean of 30 minutes. The batch is separated into four individual units (processed individually) arrived at work part B preparation area. The combined processing time for work unit B preparation is 8.5 minutes after which it is sent to the sealer. At sealer (decision) electronic components are inserted, case is assembled, sealed and then tested. The process time at sealer depends on part type i.e. 3.5 minutes for part A and 5.3 minutes for part B respectively. Approximately $90 \%$ parts passed the quality test and leave system as 'Yield Good', while $10 \%$ parts are sent to the further reworking station. At the rework stage $80 \%$ parts passed the quality (decision) and leave system as 'Yield after reworking' while remaining parts are 'scrapped'. The process is depicted in Figure 1.

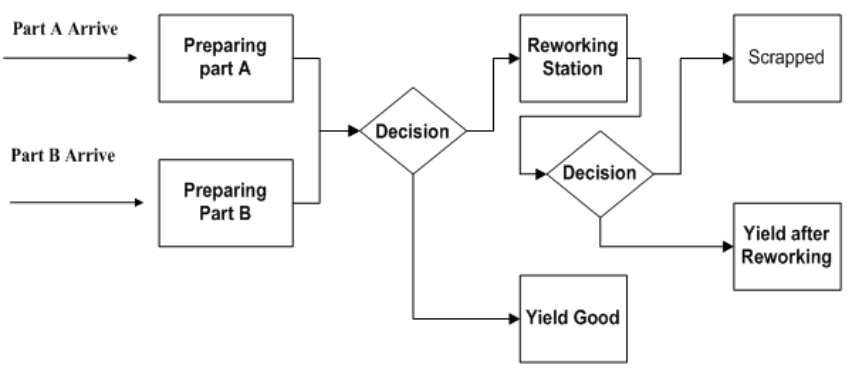

Fig. 1. The process flow of assembly line

\section{ANALYSIS AND GRAPHICAL DISPLAY}

The averaged value data has been used for the analysis. The simulation is run for 480 hours (for warm up) and replicated with different scenarios for ensuring the adequacy of the data. The experimental data is validated and verified and production runs (in simulation) are set for 12000 hours with replications. It can be argued that with finite amounts of data, it is difficult to find a specified line segment in the reconstructed phase space, therefore long range data for the research problem are selected. The parameters of interest which are affecting the performance of the assembly system are WIP, Number in Queue, Average Time in Queue, and Utilization. It is pertinent to note that WIP is sensitive in assembling process as it directly affects the system and is calculated using (2). The time persistent plots for 
the part A are shown in Figure 2. The most prominent characteristic of chaos is the unpredictability of the future regardless of deterministic time evolution. There may be an average error when forecasting the response of a future measurement which increases very rapidly with time. This unpredictability is an outcome of the inbuilt instability of the solutions, reflected by what is called sensitive dependence on initial conditions.

The time series plot for the situation as given in Figure 2 is redrawn from 250 to 350 in Figure 3 for part A. It is evident that the two trajectories are separated which shows the divergence of nearby trajectories and it is apparent that Lyapunov exponents measure the rate of divergence of initially close trajectories. The separation distance (magnitude of difference) plot is given in Figure 4 for the two time series (trajectories) data obtained in Figure 2. It is observed that the separation distance between these two trajectories varies irregularly; it indicates the sensitive dependence on initial conditions. The nature of divergence of the nearby trajectories and sensitive dependence on initial condition is quantified and characterized using the Lyapunov exponent which is computed by using the algorithm described above.

For a deterministic process, the Lyapunov exponent has to be a positive finite number and for a linear process, it should be zero. The average Lyapunov exponent of experimental data is computed to be 0.0078 as given in Fig. 5. The result obtained from the above analysis of the time series data exhibits chaotic behavior since average Lyapunov exponent is positive. The small value of average Lyapunov exponent shows presence of weak chaos but it proves that chaotic behavior may exist in flexible assembly line manufacturing systems.

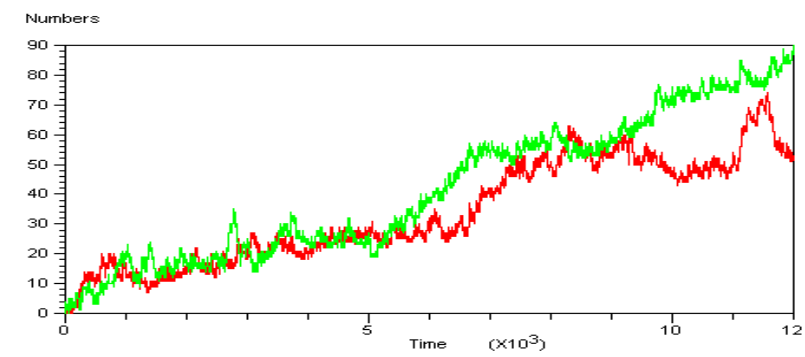

Fig. 2. Work In Process for part A

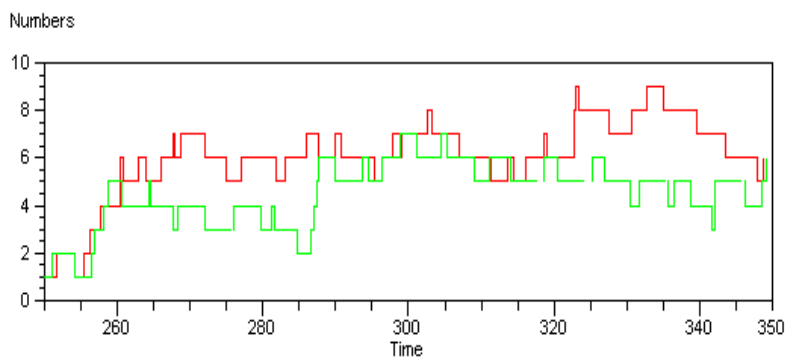

Fig. 3. Trajectories of time series data part A

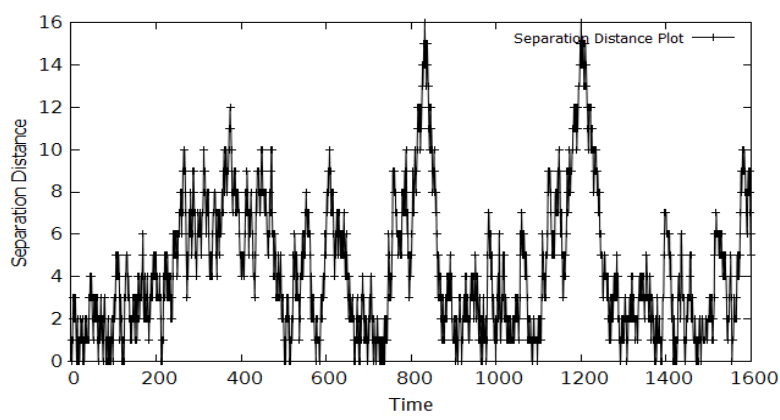

Fig. 4. Separation distance plot for WIP part A

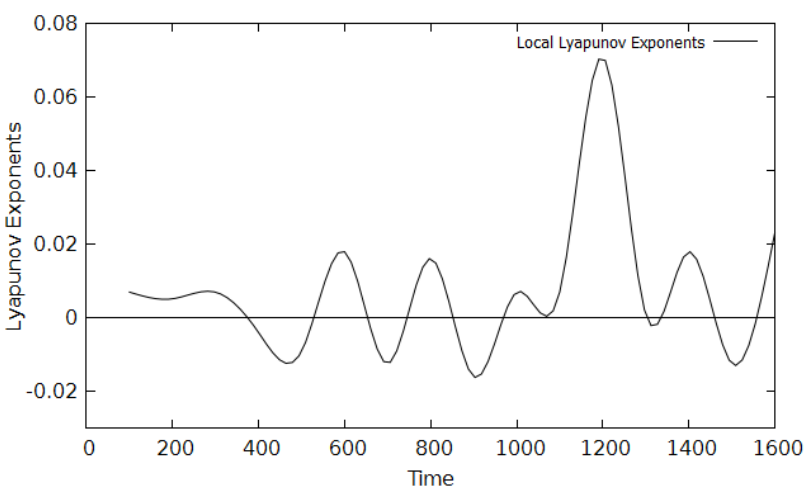

Fig. 5. Local Lyapunov exponents for WIP part A

\section{CONCLUSION}

In this work, it is concluded that flexible assembly lines may have a chaotic behavior as very small changes may lead to deviations in performance indicators such as WIP. The main interest is focused on the Lyapunov exponents since it can be calculated relatively easily and it yields confirmation of the presence of chaos in the observed data. The average Lyapunov exponent computed is positive which shows that chaotic behavior occurs in the system. It is recommended that the shop floor management must be careful when carrying out assembling with different scenarios as a chaotic behavior may result from the system. More research work is needed to investigate other similar processes and issues related to control and scheduling.

\section{REFERENCES}

[1] T. Beaumariage, K. Kempf, "The nature and origin of chaos in manufacturing systems", Proceedings of the IEEE/SEMI Advanced Semiconductor Manufacturing Conference, 169-174, 1994

[2] J. P. M. Schmitz, D. A. van Beek, J. E. Rooda, "Chaos in discrete production systems”, Journal of Manufacturing Systems, Vol. 21, No. 3, pp. 236-246, 2002

[3] B. Scholtz-Reiter, M. Freitag, A. Schmieder, "Modelling and control of production systems based on nonlinear dynamics theory”, CIRP Annals Manufacturing Technology, Vol. 51, No. 1, pp. 375-378, 2002

[4] M. D. Alfaro, J. M. Sepulveda, "Chaotic behavior in manufacturing systems”, International Journal of Production Economics, Vol. 101, pp. 150-158, 2006 
[5] N. Papakostas, D. Mourtzis, “An approach for adaptability modeling in manufacturing - analysis using chaotic dynamics”, Annals of CIRPManufacturing Technology, Vol. 56, No. 1, pp. 491-494, 2007

[6] H. A. El Maragh, M. Manns, "Transition of interarrival time patterns between automated and manual configurations of assembly systems”, Journal of Manufacturing Systems, Vol. 26, No. 1, pp. 1-11, 2007

[7] V. Toshniwal, N. Duffie, T. Jagalski, H. Rekersbrink, B. Scholz-Reiter, "Assessment of fidelity of control-theoretic models of WIP regulation in networks of autonomous work systems”, CIRP Annals - Manufacturing Technology, Vol. 60, No. 1, pp. 485-488, 2011

[8] N. Papakostas, K. Efthymiou, D. Mourtzis, G. Chryssolouris, "Modeling the complexity of manufacturing systems using non-linear dynamics approaches”, CIRP Annals - Manufacturing Technology, Vol. 58, No. 1, pp. 437-440, 2009

[9] R. Donner, B. Scholz-Reiter, U. Hinrichs, "Nonlinear characterization of the performance of production and logistics networks", Journal of Manufacturing Systems, Vol. 27, No. 2, pp. 84-99, 2008

[10] M. D. Alfaro, J. .M. Sepulveda, J. A. Ulloa, "Forecasting chaotic series in manufacturing systems by vector support machine regression and neural networks”, Int. J. Comput. Commun. Vol. 8, No. 1, pp. 8-17, 2013

[11] A. M. L. Jimenez, C. C. M. Vara De Rey, A. R. G. Torres, "Effect of parameter calculation in direct estimation of the lyapunov exponent in short time series”, Discrete Dynamics in Nature and Society, Vol. 7, No. 1, pp. 41-52, 2002

[12] Z. Liu, “Chaotic time series analysis", Mathematical Problems in Engineering. Article ID 720190, 31 pages, 2010

[13] R. C. Hilborn, Chaos and nonlinear dynamics: an introduction for scientists and engineers, $2^{\text {nd }}$ edn, Oxford University Press, Oxford, 2000

[14] J. A. Buzacott, J. G. Shanthikumar, Stochastic models of manufacturing systems, Prentice Hall, 1993

[15] F. Takens, "Detecting strange attractors in turbulence" in Dynamical Systems and Turbulence, Vol. 898, pp. 366-381, Springer Verlag, New York, 1981

[16] J. Banks, J. S. Carson, B. L. Nelson, D. M. Nicol, Discrete event system simulation, 3rd edn, Pearson Education Asia, 2002 